\title{
An interplay between attraction and repulsion in infinite populations
}

\author{
Yuri Kozitsky $^{1}$ (D)
}

Received: 9 June 2021 / Accepted: 15 July 2021 / Published online: 26 July 2021

(C) The Author(s) 2021

\begin{abstract}
We propose and study a model describing an infinite population of point entities arriving in and departing from $X=\mathbb{R}^{d}, d \geq 1$. The already existing entities force each other to leave the population (repulsion) and attract the newcomers. The evolution of the population states is obtained by solving the corresponding Fokker-Planck equation. Without interactions, the evolution preserves states in which the probability $p(n, \Lambda)$ of finding $n$ points in a compact vessel $\Lambda \subset X$ obeys the Poisson law. As we show, for pure attraction the decay of $p(n, \Lambda)$ with $n \rightarrow+\infty$ may be essentially slower. The main result is the statement that in the presence of repulsion-even of an arbitrary short range - the evolution preserves states in which the decay of $p(n, \Lambda)$ is at most Poissonian. We also derive the corresponding kinetic equation, the numerical solutions of which can provide more detailed information on the interplay between attraction and repulsion. Further possibilities in studying the proposed model are also discussed.
\end{abstract}

Keywords Fokker-Planck equation · Correlation measure · Poisson state $\cdot$ Kinetic equation

Mathematics Subject Classification 82C22 - 92D25 · 60J80

\section{Introduction}

"A system that involves numerous interacting agents whose aggregate behaviors are to be understood. Such aggregate activity is nonlinear, hence it cannot simply be derived from summation of individual components behavior". According to this typical

\footnotetext{
The present research was supported by Narodowe Centrum Nauki (NCN) Poland, Grant 2017/25/B/ST1/00051, that is cordially acknowledged by the author.

$凶 \quad$ Yuri Kozitsky

jkozi@hektor.umcs.lublin.pl

1 Instytut Matematyki, Uniwersytet Marii Curie-Skłodowskiej, 20-031 Lublin, Poland
}

Birkhäuser 
characterization, ${ }^{1}$ a large system becomes complex due to interactions between its constituents. The development of the mathematical theory of such systems is a challenging task of modern applied mathematics [2,3,31-33]. This includes also microscopic modeling populations dwelling in a continuous habitat, see $[5,7,8,18,31,33]$, in particular, those where the evolution amounts to the appearance and disappearance of their members. For such models, typical interactions are attraction and repulsion, and a key question of the theory is what might be the global outcome of their interplay.

In simple models, the population is finite and has no structure. Its state space is the set of nonnegative integers $\mathbb{N}_{0}:=\mathbb{N} \cup\{0\}$. That is, in state $n \in \mathbb{N}_{0}$ the population consists of $n$ entities, which is its complete characterization. Then the only observed result of the appearance-disappearance is the evolution of the number of entities in the population. Of course, possible interactions in this case can be taken into account only indirectly. The theory of such models goes back to works by A. Kolmogorov and W. Feller, see e.g., [1], for a more recent account of the related concepts and results. In this theory, the time evolution $t \mapsto p_{n}(t)$ of the probability that the system consists of $n$ entities is obtained by solving the Kolmogorov equation, expressed in terms of birth and death rates, $\lambda_{n}$ and $\mu_{n}$, respectively. Possible influence of the entities on each other is reflected in the dependence of theses rates on $n$. If the increase of $\lambda_{n}$ and $\mu_{n}$ is controlled by affine functions of $n$, the solution of the Kolmogorov equation is given by a stochastic semigroup, see, e.g., $[1,23]$ and the literature quoted in this work. One of the main concerns of the theory is how does the dependence of $p_{n}(t)$ on $n$ change with time.

A population becomes a complex system if it is given an inner structure closely related to interactions of its constituents-now explicitly taken into consideration. The inner structure of a given system is set by assigning a trait to each of its members. Usually, interactions are binary, dependent on the traits of the interacting entities. The set of all such traits, $X$, is mostly a locally compact topological space. It is called habitat being interpreted as the set of spatial locations of the entities. Its topology is employed to determine the (finite) collection of entities interacting with a given one. This characterizes the local structure of the population. In order to qualitatively distinguish between local and global aspects, one has to assume that $X$ is noncompact. If the whole population is finite, it is contained in a compact subset $Y \subset X$-the finite sum of compact sets containing the trait of each single entity. If the population stays in this $Y$ forever, its evolution is qualitatively 'local'. If in the course of evolution the population disperses beyond each compact subset of $X$, it can be characterized as developing. In such systems, boundary effects play a major role, see [11], whereas interactions in the bulk are not essential [24]. Thus, in order to reveal mathematical mechanisms of interaction-induced global phenomena, one has to study an infinite population dwelling in a noncompact habitat. In statistical physics, the necessity of taking an infinite-volume limit for describing a phase transition (an interaction-induced collective phenomenon) was accepted after a heated discussion followed by voting, see [38, pages 5,6]. Since that time, it has become clear [14] that the individual-based mathematical theory of an infinite complex system should employ a probabilistic/statistical apparatus-even if the elementary evolutionary acts are deterministic.

\footnotetext{
${ }^{1}$ See http://www.informatics.indiana.edu/rocha/publications/complex/csm.html
} 
In this article, we propose and study a model of an infinite population of point 'particles' (migrants), arriving in and departing from a habitat $X=\mathbb{R}^{d}, d \geq 1$. Both these acts occur at random and are described in a way that takes into account possible heterogeneity of the habitat and inter-particle attraction and repulsion (competition). It is clear, cf. [22, Sect. 2.3], that without interaction the distribution of migrants should eventually reflect the heterogeneity of the habitat and be Poissonian-in view of the randomness mentioned above and the lack of interactions. This means that the probability $p(n, \Lambda)$ of finding $n$ particles in a compact vessel $\Lambda \subset X$ obeys the Poisson law. As we show in this paper, in the case of pure attraction the decay of the occupation probabilities $p(n, \Lambda)$ with $n \rightarrow+\infty$ may be essentially slower than that in the Poissonian case, cf. (2.1) and (2.25) below. Hence, the appearance of dense clusters in the population is much more probable in such states. Assume now that the already existing population members force each other to leave (emigrate) and keep attracting the newcomers. The repulsion is effective only at short distances, whereas the attraction can be of long range. The question which we raise and answer here is whether such a short-range repulsion can restore the Poissonian decrease of the occupation probabilities $p(n, \Lambda)$. If this is the case, one can qualify it as a kind of self-regulation of the considered population

In Sect. 2 below, we introduce necessary technicalities and then provide an example of a pure attractive system where the occupation probabilities decay in a much slower way that in the Poissonian case, see (2.25). In Sect. 3, we introduce our model and discuss how does it describe the aforementioned cases of short and long repulsion, see Remark 3.2. Afterwards, we compare our model with similar models known in the literature. Next, we formulate the result-Theorem 3.9-and analyze its significance in Remark 3.10. Thereafter, we formulate and discuss Lemmas 3.12-3.15 by means of which we then prove Theorem 3.9. In subsect. 3.4, we derive the kinetic equation (3.21) corresponding to our model, the numerical study of which can provide more complex and detailed information on the interplay between attraction and repulsion in our model for studying such equations. Finally, we discuss possible continuation of studying the proposed model based on the results of the present work.

\section{The setup}

We equip the habitat $X=\mathbb{R}^{d}$ with the natural (Euclidean) distance and the corresponding mathematical structures: topology, Borel $\sigma$-field of subsets, etc. By $\mathbb{1}_{\Delta}$ we denote the indicator of a suitable set $\Delta$. For appropriate measure $\mu$ and function $f$, we write $\mu(f)=\int f d \mu$. By a Polish space we mean a separable topological space, the topology of which is consistent with a complete metric.

\subsection{Poissonian states}

The (pure) states of the population are configurations-locally finite subsets $\gamma \subset X=$ $\mathbb{R}^{d}$. The latter means that, for a given $\gamma$, the set $\gamma_{\Lambda}:=\gamma \cap \Lambda$ is finite whenever $\Lambda \subset X$ is compact. By $\Gamma$ we denote the collection of all such configurations $\gamma$. For $\Lambda \subset X$, 
one defines the counting map $\Gamma \ni \gamma \mapsto N_{\Lambda}(\gamma):=\left|\gamma_{\Lambda}\right|$, where $|\cdot|$ denotes cardinality. It takes finite values if $\Lambda$ is compact. Thereby, one sets $\Gamma^{\Lambda, n}:=\left\{\gamma \in \Gamma: N_{\Lambda}(\gamma)=n\right\}$ and equips $\Gamma$ with the $\sigma$-field $\sigma\left(\Gamma^{\Lambda, n}\right)$ generated by all $\Gamma^{\Lambda, n}, n \in \mathbb{N}_{0}$ and compact $\Lambda \subset X$. The set $\Gamma$ can also be given a topology such that the corresponding Borel $\sigma$ field $\mathcal{B}(\Gamma)$ coincides with $\sigma\left(\Gamma^{\Lambda, n}\right)$. This is the vague (weak-hash) topology [12]-the weakest topology that makes continuous all the maps $\gamma \mapsto \sum_{x \in \gamma} g(x), g \in C_{\mathrm{cs}}(X)$, where the latter is the set of all continuous compactly supported functions $g: X \rightarrow \mathbb{R}$. With this topology $\Gamma$ is a Polish space. Below, in expressions like $\gamma \cup x$ we treat $x$ as a singleton configuration $\{x\}$.

Let $\mathcal{P}(\Gamma)$ be the set of all probability measures on $(\Gamma, \mathcal{B}(\Gamma))$. They will serve as states of the population. Then, in state $\mu \in \mathcal{P}(\Gamma)$, the aforementioned occupation probability is $p(n, \Lambda)=\mu\left(\Gamma^{\Lambda, n}\right)$. Poison measures $\pi_{\chi}$ are defined by their intensity measures $\chi$-positive Radon measures of $X$, for which,

$$
\pi_{\chi}\left(\Gamma^{\Lambda, n}\right)=[\chi(\Lambda)]^{n} \exp (-\chi(\Lambda)) / n !
$$

In Poissonian states, the particles are independently distributed over the habitat, see [19, Chapter 2]. For the homogeneous Poisson measure, its intensity measure is such that $\chi(\Lambda)=\varkappa|\Lambda|$, where $|\Lambda|$ is Lebesgue's measure (Euclidean volume) of $\Lambda$ and $\varkappa>0$ is the particle density. For brevity, we write $\pi_{\varkappa}$ for such a measure, and $\pi:=\pi_{1}$.

Set $\Gamma^{n}=\{\gamma \in \Gamma:|\gamma|=n\}$. The collection of finite configurations

$$
\Gamma_{0}:=\bigcup_{n \in \mathbb{N}_{0}} \Gamma^{n}
$$

is clearly a measurable subset of $\Gamma$. In a state, $\mu$, with the property $\mu\left(\Gamma_{0}\right)=1$, the system is finite.

Proposition 2.1 The following is true: (a) if $\chi(X)=\infty$, then $\pi_{\chi}\left(\Gamma_{0}\right)=0$; (b) if $\chi(X)<\infty$, then $\pi_{\chi}\left(\Gamma_{0}\right)=1$.

Proof First, we prove the validity of (a). To this end, we take an ascending and exhausting sequence $\left\{\Lambda_{m}\right\}_{m \in \mathbb{N}}$ of compact $\Lambda_{m} \subset X$. That is, $\Lambda_{m} \subset \Lambda_{m+1}, m \in \mathbb{N}$, and each $x \in X$ is eventually contained in some $\Lambda_{m}$. It is clear that $\chi\left(\Lambda_{m}\right) \rightarrow+\infty$ in this case. Moreover, it is possible to pick $\left\{\Lambda_{m}\right\}_{m \in \mathbb{N}}$ in such a way that

$$
\sum_{m=1}^{\infty} \pi_{\chi}\left(\Gamma^{\Lambda_{m}, n}\right)<\infty
$$

see (2.1). E.g., take it such that $\chi\left(\Lambda_{m}\right) \geq \mathrm{cm}$ for some $c>0$. Assume now that $\pi_{\chi}\left(\Gamma_{0}\right)>0$. By (2.2) it then follows that $\pi_{\chi}\left(\Gamma^{n}\right)>0$ for some $n \in \mathbb{N}$. Fix this $n$ and write $\Gamma^{n}=\Gamma^{\Lambda_{m}, n} \cup \Gamma_{c}^{\Lambda_{m}, n}, \Gamma_{c}^{\Lambda_{m}, n}:=\Gamma^{n} \backslash \Gamma^{\Lambda_{m}, n}$, which makes sense for each $m$. Now for a given $\varepsilon$, we pick $m_{\varepsilon}$ such that $\chi\left(\Lambda_{m_{\varepsilon}}\right) \geq n$ and

$$
\max \left\{\pi_{\chi}\left(\Gamma^{\Lambda_{m_{\varepsilon}}, n}\right) ; \sum_{k=m_{\varepsilon}+1}^{\infty} \pi_{\chi}\left(\Gamma^{\Lambda_{k}, n}\right)\right\}<\frac{\varepsilon}{2},
$$


which is possible in view of (2.1) and (2.3). Clearly, the letter inequality remains true after replacing $m_{\varepsilon}$ by any $m>m_{\varepsilon}$. By our assumptions each $\gamma \in \Gamma^{n}$ is contained in some $\Lambda_{m}$, hence

$$
\forall m>m_{\varepsilon} \quad \Gamma_{c}^{\Lambda_{m}, n} \subset \bigcup_{k=m_{\varepsilon}+1}^{\infty} \Gamma^{\Lambda_{k}, n},
$$

by which and (2.4) we then conclude that $\pi_{\chi}\left(\Gamma^{n}\right)<\varepsilon$, which in fact yields $\pi_{\chi}\left(\Gamma_{0}\right)=$ 0 . Hence, the system in state $\pi_{\chi}$ is infinite. The proof of (b) follows directly by (2.1) with $\Lambda$ replaced by $X$.

\subsection{Sub-poissonian states}

For each $\gamma \in \Gamma$ and $m \in \mathbb{N}$, we define the following Radon measure $M_{\gamma}^{(m)}$ on $X^{m}$, see [30, page 37]. Let $x_{1}, x_{2}, \ldots$ be an enumeration of the points of $\gamma$. For a compact $\Delta \subset X^{m}$, one defines $M_{\gamma}^{(m)}(\Delta)$ as the number of ordered $m$-tuples $\left(i_{1}, \ldots, i_{m}\right)$ with distinct $i_{l}$ such that $\left(x_{i_{1}}, x_{i_{2}}, \ldots, x_{i_{m}}\right) \in \Delta$. In other words,

$$
M_{\gamma}^{(m)}(\Delta)=\sum_{x_{1} \in \gamma} \sum_{x_{2} \in \gamma \backslash x_{1}} \cdots \sum_{x_{m} \in \gamma \backslash\left\{x_{1}, \ldots, x_{m-1}\right\}} \mathbb{1}_{\Delta}\left(x_{1}, \ldots, x_{m}\right) .
$$

It is known, see [30, Theorem 1], that the map $\gamma \mapsto M_{\gamma}^{(m)}(\Delta) \in \mathbb{R}$ is measurable. However it may be unbounded. If it is $\mu$-integrable for each compact $\Delta$, then the Radon measure

$$
\rho_{\mu}^{(m)}=\int_{\Gamma} M_{\gamma}^{(m)} \mu(d \gamma)
$$

is called the $m$-th order correlation measure for this $\mu \in \mathcal{P}(\Gamma)$. If such measures exist for all $m \in \mathbb{N}$, one says that $\mu$ has finite correlations, see [30]. This is the case for $\pi_{\chi}$, where $\rho^{(m)}=\chi^{\otimes m}$, i.e., $\rho^{(m)}(\Delta)=\chi\left(\Lambda_{1}\right) \cdots \chi\left(\Lambda_{m}\right)$ for $\Delta=\Lambda_{1} \times \cdots \times \Lambda_{m}$.

Let $\theta: X \rightarrow[-1,0]$ be a measurable function that vanishes outside a compact $\Lambda$. Then the function

$$
\Gamma \ni \gamma \mapsto F^{\theta}(\gamma)=\prod_{x \in \gamma}(1+\theta(x))=\exp \left(\sum_{x \in \Lambda} \ln [1+\theta(x)]\right)
$$

is also measurable and bounded, hence $\mu$-integrable for each $\mu \in \mathcal{P}(\Gamma)$. This means that we can write

$$
\mu\left(F^{\theta}\right)=\int_{\Gamma} \exp \left(\sum_{x \in \Lambda} \ln [1+\theta(x)]\right) \mu(d \gamma)=\widehat{\mu}(\varphi), \quad \varphi(x)=-\ln [1+\theta(x)],
$$


where $\widehat{\mu}$ is the log-Laplace transform (functional) of $\mu$, cf. [13, page 43]. Now we fix $\gamma$ and $\theta$ and consider the following function

$$
\begin{aligned}
\mathbb{C} \ni z \mapsto F^{z \theta}(\gamma) & =\prod_{x \in \gamma}[1+z \theta(x)] \\
& =1+z \sum_{x \in \gamma} \theta(x)+z^{2} \sum_{x_{1} \in \gamma} \sum_{x_{2} \in \gamma \backslash x_{1}} \theta\left(x_{1} \theta\left(x_{2}\right)+\cdots,\right.
\end{aligned}
$$

which is a polynomial as $\theta$ has compact support. For $z \in[0,1], F^{z \theta}$ is $\mu$-integrable for any $\mu \in \mathcal{P}(\Gamma)$; hence, one can define

$$
f_{\theta, \mu}(z)=\mu\left(F^{z \theta}\right), \quad z \in[0,1]
$$

If $\mu$ has finite correlations, $f_{\theta, \mu}$ is infinitely differentiable at zero, and the corresponding derivatives are

$$
f_{\theta, \mu}^{(m)}(0)=\int_{X^{m}} \theta\left(x_{1}\right) \cdots \theta\left(x_{m}\right) \rho_{\mu}^{(m)}\left(d x_{1}, \ldots, d x_{m}\right), \quad m \in \mathbb{N} .
$$

In particular,

$$
f_{\theta, \pi_{\chi}}^{(m)}(0)=\left(\int_{X} \theta(x) \chi(d x)\right)^{m} .
$$

Let now $\pi_{\chi}$ in (2.11) be a homogeneous Poisson measure, i.e., $\pi_{\chi}=\pi_{\varkappa}$ for some $\varkappa>0$. Then

$$
f_{\theta, \pi_{\varkappa}}^{(m)}(0)=\varkappa^{m}\|\theta\|_{L^{1}(X)}^{m}, \quad L^{1}(X):=L^{1}(X, d x),
$$

and thus the map $\theta \mapsto f_{\theta, \pi_{\varkappa}}^{(m)}(0)$ can be continued to a continuous monomial of $\theta \in L^{1}(X)$. At the same time, for each $\theta \in L^{1}(X), z \mapsto f_{\theta, \pi_{\varkappa}}(z)$ can be continued to an exponential function of normal type.

Definition 2.2 A measure $\mu \in \mathcal{P}(\Gamma)$ is called sub-Poissonian if, for each $\theta \in L^{1}(X)$, the function $f_{\theta, \mu}$ defined in (2.9) can be continued to an exponential function of normal type. The set of all such measures will be denoted by $\mathcal{P}_{\text {exp }}$.

For each $\mu \in \mathcal{P}_{\text {exp }}$, it follows that

$$
f_{\theta, \mu}^{(m)}(0)=\int_{X^{m}} k_{\mu}^{(m)}\left(x_{1}, \ldots x_{m}\right) \theta\left(x_{1}\right) \cdots \theta\left(x_{m}\right) d x_{1} \cdots d x_{m}
$$

where $k_{\mu}^{(m)}$ is a symmetric element of $L^{\infty}\left(X^{m}\right)$, called $m$-th order correlation function of $\mu$, see [30,37], satisfying

$$
0 \leq k_{\mu}^{(m)}\left(x_{1}, \ldots x_{m}\right) \leq \varkappa^{m}
$$


Here symmetric means $k_{\mu}^{(m)}\left(x_{1}, \ldots x_{m}\right)=k_{\mu}^{(m)}\left(x_{\sigma(1)}, \ldots x_{\sigma(m)}\right)$, holding for all permutations $\sigma \in \Sigma_{m}$. The least $\varkappa$ such that (2.13) holds for a given $\mu \in \mathcal{P}_{\exp }$ is called the type of $\mu$. The estimate in (2.13) is known as Ruelle's bound. States of thermal equilibrium of infinite systems of interacting point particles placed in $\mathbb{R}^{d}$ have correlation functions satisfying (2.13), see [37].

Remark 2.3 According to Definition 2.2, sub-Poissonian measures have finite correlations, and their correlation measures are absolutely continuous with respect to the corresponding Lebesgue measures. This applies also to Poisson measures, i.e., $\pi_{\chi} \in \mathcal{P}_{\exp }$ if and only if $\chi(d x)=q(x) d x$ with $q \in L^{\infty}(X)$. In this case,

$$
k_{\pi_{\chi}}^{(m)}\left(x_{1}, \ldots, x_{m}\right)=q\left(x_{1}\right) \cdots q\left(x_{m}\right), \quad m \in \mathbb{N} .
$$

For $m \in \mathbb{N}$, let $G^{(m)}: X^{m} \rightarrow \mathbb{R}$ be a measurable symmetric function, bounded and vanishing outside a compact $\Delta$. Then as in (2.5) we have

$$
\begin{aligned}
M_{\gamma}^{(m)}\left(G^{(m)}\right) & =\sum_{x_{1} \in \gamma} \sum_{x_{2} \in \gamma \backslash x_{1}} \ldots \sum_{x_{m} \in \gamma \backslash\left\{x_{1}, \ldots, x_{m-1}\right\}} G^{(m)}\left(x_{1}, \ldots, x_{m}\right) \\
& =:\left(K G^{(m)}\right)(\gamma),
\end{aligned}
$$

which determines a map on the symmetric part of $B_{\mathrm{cs}}\left(X^{m}\right)$-the set of all bounded measurable functions, each vanishing outside its own compact support. By (2.5) and (2.6) we have

$$
\mu\left(K G^{(m)}\right)=\rho_{\mu}^{(m)}\left(G^{(m)}\right)=\int_{X^{m}} k_{\mu}^{(m)}\left(x_{1}, \ldots, x_{m}\right) G^{(m)}\left(x_{1}, \ldots, x_{m}\right) d x_{1} \cdots d x_{m} .
$$

If $G^{(m)} \geq 0$ and $\mu \in \mathcal{P}_{\text {exp }}$, by (2.16) and (2.13) it follows that $\mu\left(K G^{(m)}\right) \leq$ $\varkappa^{m}\left\|G^{(m)}\right\|_{L^{1}\left(X^{m}\right)}=\pi_{\varkappa}\left(K G^{(m)}\right)$, where $\varkappa$ is the type of $\mu$. In this sense, $\mu$ is indeed sub-Poissonian.

Let $G$ denote a sequence $\left\{G^{(m)}\right\}_{m \in \mathbb{N}_{0}}$ of symmetric $G^{(m)} \in B_{\mathrm{cs}}\left(X^{m}\right)$. Here $G^{(0)}$ is just a real number. Let then $\mathcal{G}^{0}$ be the set of all such sequences with the property: $G^{(m)} \equiv 0$ whenever $m>m_{G}$ with $G$-specific $m_{G} \in \mathbb{N}$. In a natural way, one equips $\mathcal{G}^{0}$ with the pointwise linear operations and multiplication, and then sets

$$
K G=\sum_{m \geq 0} \frac{1}{m !} K G^{(m)}, \quad G \in \mathcal{G}^{0} .
$$

Note that each $G$ gives rise to a function $G: \Gamma_{0} \rightarrow \mathbb{R}$, see (2.2), such that $G(\gamma)=$ $G^{(m)}\left(x_{1}, \ldots, x_{m}\right)$ for $\gamma=\left\{x_{1}, \ldots, x_{m}\right\}$. A particular case of such a function is

$$
e(\theta ; \gamma)=\prod_{x \in \gamma} \theta(x), \quad e(\theta ; \varnothing) \equiv 1
$$


where $\theta$ is a measurable function. For $\theta \in B_{\mathrm{bs}}(X)$, we have, cf. (2.17) and (2.15),

$$
(K e(\theta ; \cdot))(\gamma)=1+\sum_{x \in \gamma} \theta(x)+\sum_{x_{1} \in \gamma} \sum_{x_{2} \in \gamma \backslash x_{1}} \theta\left(x_{1}\right) \theta\left(x_{2}\right)+\cdots=F^{\theta}(\gamma)
$$

For $G \in \mathcal{G}^{0}$, let $C_{G}$ be such that

$$
\left|G^{(m)}\left(x_{1}, \ldots, x_{m}\right)\right| \leq C_{G}
$$

holding for each $m$ and all $\left(x_{1}, \ldots, x_{m}\right) \in X^{m}$. Let also $\Lambda_{G}$ be such that $G^{(m)}\left(x_{1}, \ldots, x_{m}\right)=0$ whenever either of $x_{j}$ is in $X \backslash \Lambda_{G}$. Then (2.17) implies

$$
|(K G)(\gamma)| \leq C_{G}\left(1+N_{\Lambda_{G}}(\gamma)\right)^{m_{G}} .
$$

In view of the latter estimate, let us calculate $\mu\left(N_{\Lambda}^{m}\right)$. By standard combinatorial formulas it follows that

$$
\left[N_{\Lambda}(\gamma)\right]^{m}=\sum_{l=1}^{m} l ! S(m, l) M_{\gamma}^{(l)}\left(\Lambda^{l}\right)
$$

where $M_{\Lambda}^{(l)}$ is defined in (2.5) and $S(m, l)>0$ are Stirling's numbers of the second kind: $S(m . l)$ is the number of ways to divide $m$ labeled items into $l$ unlabeled groups, see [36, Chapter 2] or [9, Sect. 3]. By (2.6) we then have

$$
\mu\left(N_{\Lambda}^{m}\right)=\sum_{l=1}^{m} l ! S(m, l) \rho_{\mu}^{(l)}\left(\Lambda^{l}\right)
$$

For $\mu \in \mathcal{P}_{\text {exp }}$, let $k_{\mu}$ stand for the sequence $\left\{k_{\mu}^{(m)}\right\}_{m \in \mathbb{N}_{0}}$, where $k_{\mu}^{(0)}=1$ and $k_{\mu}^{(m)}$, $m \geq 1$ are as in (2.12). Then by (2.16) and (2.17) we get

$$
\begin{aligned}
\mu(K G) & =G^{(0)}+\sum_{m=1}^{\infty} \frac{1}{m !} \int_{X^{m}} k_{\mu}^{(m)}\left(x_{1}, \ldots, x_{m}\right) G^{(m)}\left(x_{1}, \ldots, x_{m}\right) d x_{1} \cdots d x_{m} \\
& =\int_{\Gamma_{0}} k_{\mu}(\gamma) G(\gamma) \lambda(d \gamma)=:\left\langle\left\langle k_{\mu}, G\right\rangle,\right.
\end{aligned}
$$

where $\lambda$-the Lebesgue-Poisson measure-is a Borel measure on $\left(\Gamma_{0}, \mathcal{B}\left(\Gamma_{0}\right)\right)$, determined by the integrals

$$
\int_{\Gamma_{0}} G(\gamma) \lambda(d \gamma)=G^{(0)}+\sum_{m=1}^{\infty} \frac{1}{m !} \int_{X^{m}} G^{(m)}\left(x_{1}, \ldots, x_{m}\right) d x_{1} \cdots d x_{m}, \quad G \in \mathcal{G}^{0} .
$$


It is clear that such integrals determine the measure, cf. Proposition 3.3 below. These integrals have the following useful property

$$
\int_{\Gamma_{0}}\left(\int_{X} H(x, \gamma) d x\right) \lambda(d \gamma)=\int_{\Gamma_{0}}\left(\sum_{x \in \gamma} H(x, \gamma \backslash x)\right) \lambda(d \gamma)
$$

holding for all appropriate functions $H$, see [25, Proposition 1].

\subsection{Clustering}

Assume now that a given $\mu \in \mathcal{P}(\Gamma)$ is such that $\mu\left(N_{\Lambda}\right)<\infty$ for each compact $\Lambda \subset X$. Then the values of $\mu\left(\Gamma^{\Lambda, n}\right), n \in \mathbb{N}_{0}$, can be compared with those for $\pi_{\chi}$ with $\chi(\Lambda)=\mu\left(N_{\Lambda}\right)=\rho_{\mu}^{(1)}(\Lambda)$, i.e., $\chi=\rho_{\mu}^{(1)}$, see (2.6). For sub-Poissonian measures, the function (2.9) makes sense for all $\theta \in L^{1}(X)$, in particular, for $\theta=\mathbb{1}_{\Lambda}$ for each compact $\Lambda$. Then by (2.12) and (2.13) we have

$$
\mu\left(\Gamma^{\Lambda, n}\right)=\frac{1}{n !} f_{\theta, \mu}^{(n)}(-1)=\frac{1}{n !} \sum_{m=0}^{\infty} \frac{(-1)^{m}}{m !} \rho_{\mu}^{(n+m)}\left(\Lambda^{n+m}\right) \leq \frac{1}{n !}(\varkappa|\Lambda|)^{n} e^{\varkappa|\Lambda|},
$$

where $\varkappa>0$ is the type of $\mu$. This means that the asymptotic of $\mu\left(\Gamma^{\Lambda, n}\right)$ is at most the same as in (2.1) with $\mu\left(N_{\Lambda}\right)=\varkappa|\Lambda|$, i.e., the following holds

$$
\frac{\mu\left(\Gamma^{\Lambda, n}\right)}{\pi_{\chi}\left(\Gamma^{\Lambda, n}\right)} \rightarrow 0, \quad n \rightarrow+\infty
$$

whenever $\varkappa|\Lambda|<\chi(\Lambda)$.

Remark 2.4 If $\mu \in \mathcal{P}(\Gamma)$ has finite correlations, a more direct way of analyzing the corresponding large occupation asymptotic is based on the use of its correlation measures. Recall that $\rho_{\mu}^{(n)}\left(\Lambda^{n}\right) / n$ ! is the $\mu$-expected number of $n$-element groups (clusters) of migrants contained in $\Lambda$, see (2.6). In the sub-Poissonian case, we have that this number is bounded by $(\varkappa|\Lambda|)^{n} / n$ !, which we have used in (2.21). That is, clustering in a state $\mu$ can be detected by establishing that (2.22) fails to hold for some $\chi$ and $\Lambda$ satisfying $\mu\left(N_{\Lambda}\right)<\chi(\Lambda)$. For states that have finite correlations, clustering occurs if the sequence $\left\{\rho_{\mu}^{(n)}\left(\Lambda^{n}\right) /[\varkappa|\Lambda|)^{n}\right\}_{n \in \mathbb{N}}$ is unbounded for all $\varkappa>0$ and some compact $\Lambda \subset X$. The lack of clustering in sub-Poisson states, that occurs due to the repulsion (competition), can also be interpreted as a kind of self-regulation [21,28].

Let us now consider the following example. By (2.11) and (2.8) we have that

$$
\pi_{\chi}\left(F^{\theta}\right)=\exp (\chi(\theta))=\exp \left(\int_{X} \theta(x) \chi(d x)\right) .
$$


Let now $\chi$ in (2.23) be random and $v$ its law. For instance, points of $\gamma$ are attracted by 'attraction centers' that constitute a configuration $\omega \in \Gamma$. In this case, we have

$$
\int_{X} \theta(x) \chi(d x)=\int_{X} \theta(x) \chi_{\omega}(d x)=\sum_{x \in \omega} \theta(x),
$$

and the joint distribution of $\gamma$ and $\omega$ is a probability measure on $\Gamma \times \Gamma$. For its marginal $Q(d \gamma)$, we then have, see (2.23) and (2.8),

$$
\begin{aligned}
Q\left(F^{\theta}\right) & =\int_{\Gamma} \int_{\Gamma} F^{\theta}(\gamma) \pi_{\chi_{\omega}}(d \gamma) \nu(d \omega) \\
& =\int_{\Gamma} \exp \left(\int_{X} \theta(x) \chi_{\omega}(d x)\right) \nu(d \omega)=\widehat{v}(-\theta) .
\end{aligned}
$$

For $v=\pi, Q$ is an analog of the Cox cluster measure, see [13, page 46]. In this case, for $\theta=\mathbb{1}_{\Lambda}$, by (2.24) we obtain

$$
f_{\theta, Q}(z)=Q\left(F^{z \theta}\right)=\exp \left(\int_{X}\left[e^{z \theta(x)}-1\right] d x\right)=\exp \left(\left[e^{z}-1\right]|\Lambda|\right) .
$$

Hence, $Q\left(N_{\Lambda}\right)=f_{\theta, Q}^{\prime}(0)=|\Lambda|$, that coincides with $\pi\left(N_{\Lambda}\right)=|\Lambda|$. To find $Q\left(\Gamma^{\Lambda, n}\right)$ we use (2.21), which means that we have to calculate $f_{\theta, Q}^{(n)}(-1)$ with such $\theta$. By standard formulas, see [9, Eq. (2.19), page 5], we get

$$
f_{\theta, Q}^{(n)}(-1)=C_{\Lambda} \phi_{n}\left(c_{\Lambda}\right), \quad c_{\Lambda}=e^{-1}|\Lambda|, \quad C_{\Lambda}=\exp \left(c_{\Lambda}-|\Lambda|\right),
$$

where $\phi_{n}(x), n \in \mathbb{N}$, are polynomials attributed to J. A. Grunert, S. Ramanujan, J. Touchard, and others, see [9, page 6] and/or [36, Chapter 2]. For $x \geq 1$, they satisfy $\phi_{n}(x) \geq x B_{n}$, where the latter is Bell's number. The large $n$ asymptotic of $B_{n}$ is known [10, page 108], by means of which we conclude that

$$
Q\left(\Gamma^{\Lambda, n}\right) \geq c_{\Lambda} C_{\Lambda} B_{n} / n ! \geq \frac{c_{\Lambda} C_{\Lambda}}{\sqrt{2 \pi}(\ln n)^{n}}
$$

As $n \rightarrow+\infty$, the right-hand side of (2.25) decays to zero in a much slower way than the right-hand side of (2.1), even if $Q\left(N_{\Lambda}\right)<|\Lambda|$, cf. (2.22). 


\section{The result}

\subsection{The model}

The stochastic evolution of states $\mu_{0} \rightarrow \mu_{t}$ is obtained by solving the Fokker-Planck equation

$$
\mu_{t}(F)=\mu_{0}(F)+\int_{0}^{t} \mu_{s}(L F) d s,
$$

in which $L$ is the model-specific Kolmogorov operator acting on test functions $F$ : $\Gamma \rightarrow \mathbb{R}$ taken from a sufficiently big class. We refer the reader to the monograph [6] for a general theory of Fokker-Planck equations. The model we propose and study in this work is specified by the following operator

$$
\begin{aligned}
(L F)(\gamma)= & \int_{\mathbb{R}^{d}} E^{+}(x, \gamma)[F(\gamma \cup x)-F(\gamma)] d x \\
& -\sum_{x \in \gamma} E^{-}(x, \gamma \backslash x[F(\gamma)-F(\gamma \backslash x)] .
\end{aligned}
$$

The first term in (3.2) describes immigration with rate

$$
E^{+}(x, \gamma)=b^{+}(x)+\sum_{y \in \gamma} a^{+}(x-y)
$$

where $b^{+}$and $a^{+}$are nonnegative. Here the first term corresponds to state-independent immigration, whereas the second one describes attraction of the arriving 'immigrants' by the already existing population. The second term in (3.2) describes emigration. Similarly as in (3.3), we take it in the form

$$
E^{-}(x, \gamma)=b^{-}(x)+\sum_{y \in \gamma} a^{-}(x-y)
$$

where both $b^{-}$and $a^{-}$are nonnegative. Note that the second term in (3.4) describes repulsion of the 'particle' located at $x$ by the rest of the population, that can also be considered as competition. The dependence of $b^{ \pm}$on $x \in X$ reflects possible heterogeneity of the habitat. In the sequel, the functions $a^{ \pm}$and $b^{ \pm}$are called kernels. The probabilistic meaning of both terms in (3.2) is as follows. For a given $\gamma$ and $x \in \gamma$, the probability of finding a particle at $x$ after time $t$ is $\exp \left(-t E^{-}(x, \gamma \backslash x)\right)$. Likewise, the probability to find a new particle at $y \in \mathbb{R}^{d}$ after time $t$ is $1-\exp \left(-t E^{+}(y, \gamma)\right)$, where the difference in these two formulas is related to the different signs of the corresponding terms of $L$ and differen initial conditions.

Assumption 3.1 The kernels $a^{ \pm}$in (3.3) and (3.4) are continuous and belong to $L^{1}\left(\mathbb{R}^{d}\right) \cap L^{\infty}\left(\mathbb{R}^{d}\right)$. Additionally, we assume that $a^{ \pm}(x)=a^{ \pm}(-x)$ and $a^{-}(0)>0$. The latter means that $a^{-}(x) \geq a_{*}^{-}>0$ if $|x| \leq r_{*}$, holding for some $a_{*}^{-}$and $r_{*}>0$. The kernels $b^{ \pm}$are continuous and bounded. 
According to this we set

$$
\left\|a^{ \pm}\right\|=\sup _{x \in \mathbb{R}^{d}} a^{ \pm}(x), \quad\left\|b^{ \pm}\right\|=\sup _{x \in \mathbb{R}^{d}} b^{ \pm}(x) .
$$

Remark 3.2 Concerning the kernels $a^{ \pm}$, the following alternatives are possible:

(i) (long repulsion) there exists $\vartheta>0$ such that $a^{-}(x) \geq \vartheta a^{+}(x)$ for all $x \in \mathbb{R}^{d}$;

(ii) (short repulsion) for each $\vartheta>0$, there exists $x \in \mathbb{R}^{d}$ such that $a^{-}(x)<\vartheta a^{+}(x)$.

In case (i), $a^{+}$decays faster than $a^{-}$, and hence the effect of repulsion from the existing population prevails. If $b^{+}(x) \equiv 0$, new members appear only due to the existing population, which can also be interpreted as their birth. In this case, $a^{+}$is usually called dispersal kernel and (i) corresponds to short dispersal. This particular case of (3.2) with nonzero $b^{-}$and $a^{-}$is the Bolker-Pacala model-introduced in [7,8] and studied in $[17,20,34]$. Models with short dispersal are employed to describe, e.g., the evolution of cell communities. An instance of the long repulsion (short dispersal) is given by $a^{+}$with finite range, i.e., $a^{+}(x) \equiv 0$ for all $|x| \geq r$, and $a^{-}(x)>0$ for such $x$. In case (ii), $a^{-}$decays faster than $a^{+}$, and hence some of the newcomers can be out of the competitive influence of the existing population. At the same time they are attracted by the latter. Models of this kind can be adequate, e.g., in plant ecology with the long-range dispersal of seeds, cf. [33]. Notably, the equality $a^{+}=a^{-}$ corresponding to case (i)-does not yet mean the lack of interaction. This is a typical example of 'nonlinearity' of a complex system mentioned above.

Particular cases of the model specified by (3.2) were studied in: (a) [17,20], case of $b^{+} \equiv 0$; (b) [22], case of $a^{+} \equiv 0$. For $b^{+} \equiv 0$, our result-formulated in Theorem 3.9 below-yields an extension of the corresponding result of [20] as it holds for both cases, (i) and (ii), mentioned in Remark 3.2. For $b^{+} \equiv 0$ and $a^{-} \equiv 0$, the model becomes exactly soluble. It is known as the continuum contact model. Under the condition

$$
\inf _{x:|x| \leq r} a^{+}(x) \geq a_{*}^{+}>0
$$

holding for some $r>0$ and $a_{*}^{+}$, cf. Assumption 3.1, for this model it was proved [16, Eq. (3.5), page 303] that

$$
k_{\mu_{t}}^{(n)}\left(x_{1}, \ldots, x_{n}\right) \geq \omega^{n t} n !
$$

for some $\omega>0$, all $n \geq 2$ and $t>0$, and almost all $x_{1}, \ldots, x_{n}$ satisfying $\left|x_{i}-x_{j}\right| \leq r$, $i, j=1, \ldots, n$. Hence, for $\Lambda$ with diameter in $X$ that does not exceed $r$, this implies, cf. Remark 2.4,

$$
\frac{1}{n !} \rho_{\mu_{t}}^{(n)}\left(\Lambda^{n}\right) \geq\left[\omega^{t}|\Lambda|\right]^{n} \rightarrow+\infty, \quad n \rightarrow+\infty
$$

holding for sufficiently large $t$. It is possible to show that a similar bound holds true also in the model described by $L$ as in (3.2) with $a^{-} \equiv 0$ and nonzero $b^{+}$. This means 
that the inter-particle competition represented in $L$ by $a^{-}$-that gives rise to the increase of emigration-is the sole factor responsible for the effect mentioned in Remark 3.10 below. Likewise, by comparing with the Bolker-Pacala model obtained from the letter by setting $b^{+} \equiv 0$, one shows that if the following holds

$$
\inf _{x \in X} b^{-}(x) \geq \int_{X} a^{+}(x) d x
$$

then the correlation functions $k_{\mu_{t}}$ remain bounded in time, see [20]. That is, the global regulation may be achieved at the expense of large emigration, see [21,28]. Moreover, the system eventually gets empty in this case.

\subsection{The domain of $L$}

In order to define the very notion of a solution of (3.1), one has to establish the domain of the Kolmogorov operator $L$. It should be: (i) big enough to identify measures by their valued $\mu(F)$ on its elements; (ii) suitable to deal with. The reason from item (i) is taken into account as follows. A set of measurable functions, $\mathcal{F}$, is called separating for $\mathcal{P}(\Gamma)$ if $\mu_{1}(F)=\mu_{2}(F)$ holding for all $F \in \mathcal{F}$ implies $\mu_{1}=\mu_{2}$ for each pair $\mu_{1}, \mu_{2} \in \mathcal{P}(\Gamma)$. Since $\Gamma$ is a Polish space, we can employ here $[15$, Theorem 4.4.5, page 113], according to which $\mathcal{D}(L)$ should contain a subset, $\mathcal{F}$, possessing the following properties: (a) $1 \in \mathcal{F}$; (b) pointwise product $F_{1} F_{2}$ should be in $\mathcal{F}$ for $F_{1}, F_{2} \in \mathcal{F}$; (c) for each pair of distinct $\gamma, \gamma^{\prime} \in \Gamma$, one finds $F \in \mathcal{F}$ such that $F(\gamma) \neq F\left(\gamma^{\prime}\right)$.

Proposition 3.3 Let $\mathcal{F}$ be the set of all $F^{\theta}$ as in (2.7) with $\theta \in C_{\mathrm{cs}}(X \rightarrow[-1,0])$, the latter being the set of all continuous compactly supported functions $\theta: X \rightarrow[-1,0]$. Then $\mathcal{F}$ is separating.

Proof With the properties just mentioned, the situation is as follows. To get (a) one takes $\theta \equiv 0$; (b) follows by the fact that $1+\theta$ is a continuous compactly supported function with values in $[0,1]$, and the product of two such functions is also of this kind. To get (c) one takes $\theta$ such that $\theta(x)=0$ for all $x \in \gamma$, and $\theta(x)=-1$ for $x \in \gamma^{\prime} \backslash \gamma$, which is possible by Uryson's lemma as each $\gamma$ has no accumulation point $x \in X$.

To take into account (ii), we use (2.19), i.e., present $F$ as $K G$, see (2.17), and then pass the action of $L$ to $G$. Note that $F^{\theta}=K e(\theta ; \cdot)$, see (2.18).

Proposition 3.4 For each $G \in \mathcal{G}^{0}$, the following is true: $(L K G)(\gamma)=(K \widehat{L} G)(\gamma)$, $\gamma \in \Gamma$, with

$$
\begin{aligned}
(\widehat{L} G)(\gamma)= & \int_{X} E^{+}(x, \gamma) G(\gamma \cup x) d x+\sum_{x \in \gamma} \int_{X} a^{+}(x-y) G(\gamma \backslash x \cup y) d y \\
& -\left(\sum_{x \in \gamma} E^{-}(x, \gamma \backslash x)\right) G(\gamma)-\sum_{x \in \gamma}\left(\sum_{y \in \gamma \backslash x} a^{-}(x-y)\right) G(\gamma \backslash x)
\end{aligned}
$$




$$
=:\left(A_{1} G\right)(\gamma)+\cdots+\left(A_{4} G\right)(\gamma)
$$

Proof By (2.15) and (2.17) for $F=K G$ we have

$$
F(\gamma)=\sum_{\xi \subset \gamma} G(\xi), \quad F(\gamma \cup y)-F(\gamma)=\sum_{\xi \subset \gamma} G(\xi \cup y), \quad y \in X .
$$

Let $L^{+}$be given by the first summand in (3.2). Then

$$
\begin{aligned}
\left(L^{+} F\right)(\gamma)= & \int_{X} E^{+}(\gamma, y) \sum_{\xi \subset \gamma} G(\xi \cup y) d y=\sum_{\xi \subset \gamma} \int_{X} b^{+}(y) G(\xi \cup y) d y \\
& +\sum_{\xi \subset \gamma} \int_{X}\left(\sum_{x \in \xi} a^{+}(x-y)+\sum_{x \in \gamma \backslash \xi} a^{+}(x-y)\right) G(\xi \cup y) d y \\
= & \sum_{\xi \subset \gamma}\left(\int_{X} E^{+}(\xi, y) G(\xi \cup y) d y+\int_{X} \sum_{x \in \xi} a^{+}(x-y) G(\xi \backslash x \cup y) d y\right) .
\end{aligned}
$$

To get the latter line we did the following calculations

$$
\begin{aligned}
\sum_{\xi \subset \gamma} \sum_{x \in \gamma \backslash \xi} a^{+}(x-y) G(\xi \cup y) & =\sum_{x \in \gamma} \sum_{\xi \subset \gamma \backslash x} a^{+}(x-y) G(\xi \cup y) \\
& =\sum_{\xi \subset \gamma} \sum_{x \in \xi} a^{+}(x-y) G(\xi \backslash x \cup y) .
\end{aligned}
$$

Similarly, for the second line in (3.2) we get

$$
\begin{aligned}
\left(L^{-} F\right)(\gamma)= & -\sum_{x \in \gamma} E^{-}(x, \gamma \backslash x)[F(\gamma)-F(\gamma \backslash x)] \\
= & -\sum_{x \in \gamma} \sum_{\xi \subset \gamma \backslash x} b^{-}(x) G(\xi \cup x)-\sum_{x \in \gamma} \sum_{\xi \subset \gamma \backslash x} G(\xi \cup x) \\
& \left(\sum_{y \in \xi} a^{-}(x-y)+\sum_{y \in \gamma \backslash(\xi \cup x)} a^{-}(x-y)\right) \\
= & -\sum_{x \in \gamma} \sum_{\xi \subset \gamma \backslash x} E^{-}(x, \xi) G(\xi \cup x)-\sum_{x \in \gamma} \sum_{\xi \subset \gamma \backslash x} G(\xi \cup x) \sum_{y \in \gamma \backslash(\xi \cup x)} a^{-}(x-y) \\
= & -\sum_{\xi \subset \gamma}\left(\sum_{x \in \xi} E^{-}(x, \xi \backslash x)\right) G(\xi)-\sum_{\xi \subset \gamma} G(\xi) \sum_{y \in \gamma \backslash \xi} \sum_{x \in \xi} a^{-}(x-y)
\end{aligned}
$$




$$
=-\sum_{\xi \subset \gamma}\left(\sum_{x \in \xi} E^{-}(x, \xi \backslash x)\right) G(\xi)-\sum_{\xi \subset \gamma} \sum_{x \in \xi} G(\xi \backslash x) \sum_{y \in \xi \backslash x} a^{-}(x-y) .
$$

Now by (3.7) and (3.8) we get the equality in question.

Now we introduce extensions of $\mathcal{G}^{0}$.

Definition 3.5 Let $C>0$ and $G=\left\{G^{(m)}\right\}_{m \in \mathbb{N}_{0}}$ be such that each $G^{(m)}$ is a symmetric element of $L^{1}\left(X^{m}\right)$. Then the set $\mathcal{G}_{C}$ consists of all those $G=\left\{G^{(m)}\right\}_{m \in \mathbb{N}_{0}}$ for which the following holds

$$
\|G\|_{C}:=\left|G^{(0)}\right|+\sum_{m=1}^{\infty} \frac{C^{m}}{m !}\left\|G^{(m)}\right\|_{L^{1}\left(X^{m}\right)}<\infty .
$$

We also set $\mathcal{G}=\cap_{C>0} \mathcal{G}_{C}$.

Obviously, each $\mathcal{G}_{C}, C>0$, equipped with the mentioned linear operations and $\|\cdot\|_{C}$ defined above is a real Banach space. Note that

$$
\|G\|_{C}=\int_{\Gamma_{0}} C^{|\gamma|}|G(\gamma)| \lambda(d \gamma)
$$

and

$$
\|e(\theta ; \cdot)\|_{C}=1+\sum_{n=1}^{\infty} \frac{1}{n !}(C\|\theta\|)^{n}, \quad\|\theta\|:=\|\theta\|_{L^{1}(X)},
$$

which means that $e(\theta ; \cdot) \in \mathcal{G}$ whenever $\theta \in L^{1}(X)$. The proof of the next statement follows directly by $(2.5)$.

Proposition 3.6 Let $\mu \in \mathcal{P}_{\exp }$ be of type $\varkappa$, see Definition 2.2 and (2.13). Then the map $\Gamma \ni \gamma \mapsto(K G)(\gamma)$ is $\mu$-integrable whenever $G \in \mathcal{G}_{C}$ with $C \geq \varkappa$.

Now we set

$$
\mathcal{D}(L)=\{F=K G: G \in \mathcal{G}\} .
$$

Proposition 3.7 For each $F \in \mathcal{D}(L)$, it follows that $L F \in \mathcal{D}(L)$.

Proof In view of (3.9) and Proposition 3.4, the proof can be done if we show that $\widehat{L}: \mathcal{G}_{C} \rightarrow \mathcal{G}_{C+\varepsilon}$, holding for all positive $C$ and $\varepsilon$. By means of (2.20) and (3.5) we 
get

$$
\left|\sum_{x \in \eta} E^{ \pm}(x, \eta)\right| \leq|\eta|\left(\left\|b^{ \pm}\right\|+\frac{1}{2}\left\|a^{ \pm}\right\|(|\eta|-1)\right)=: c^{ \pm}(|\eta|), \quad \eta \in \Gamma_{0} .
$$

Thereby, we show that each $A_{i}$ defined in the last line of (3.6) acts as a bounded linear operator from $\mathcal{G}_{C}$ to $\mathcal{G}_{C+\varepsilon}$, that holds for each positive $C$ and $\varepsilon$. By (3.10) we get

$$
\sup _{n \in \mathbb{N}} c^{ \pm}(n)\left(\frac{C}{C+\varepsilon}\right)^{n}=: \delta^{ \pm}(C, \varepsilon)<\infty
$$

Then by means of (2.20) it follows that

$$
\begin{aligned}
\left\|A_{1} G\right\|_{C} & \leq \int_{\Gamma_{0}}\left(\int_{X}\left|E^{+}(x, \eta)\right||G(\eta \cup x)| d x\right) C^{|\eta|} \lambda(d \eta) \\
& =\int_{\Gamma_{0}}\left|\sum_{x \in \eta} E^{+}(x, \eta \backslash x)\right||G(\eta)| C^{|\eta|-1} \lambda(d \eta) \\
& \leq C^{-1} \delta^{+}(C, \varepsilon)\|G\|_{C+\varepsilon} .
\end{aligned}
$$

In a similar way, we get

$$
\begin{aligned}
\left\|A_{2} G\right\|_{C} & \leq \delta^{+}(C, \varepsilon)\|G\|_{C+\varepsilon} \\
\left\|A_{3} G\right\|_{C} & \leq \delta^{-}(C, \varepsilon)\|G\|_{C+\varepsilon} \\
\left\|A_{4} G\right\|_{C} & \leq C \delta^{-}(C, \varepsilon)\|G\|_{C+\varepsilon}
\end{aligned}
$$

In combination with (3.11) this implies that $\widehat{L}$ acts as a bounded linear operator from each $\mathcal{G}_{C}$ to $\mathcal{G}_{C+\varepsilon}$, which yields the proof.

\subsection{The statement}

Now we are at a position to formulate our main statement.

Definition 3.8 By a solution of the Fokker-Planck equation (3.1) we understand a map $[0,+\infty) \ni t \mapsto \mu_{t} \in \mathcal{P}(\Gamma)$ such that: (a) each $F \in \mathcal{D}(L)$, see (3.9), is $\mu_{t}$-absolutely integrable for all $t \geq 0$; (b) for each $F \in \mathcal{D}(L)$, the map $[0,+\infty) \ni t \mapsto \mu_{t}(L F) \in \mathbb{R}$ is integrable on each $[0, T], T>0$ and (3.1) is satisfied.

According to this definition, if $t \mapsto \mu_{t}$ is a solution, then $t \mapsto \mu_{t}(F) \in \mathbb{R}$ is absolutely continuous, and hence $\mu_{t}(F) \rightarrow \mu_{0}(F)$ as $t \rightarrow 0$, where $\mu_{0}$ is considered as the initial condition for (3.1).

Theorem 3.9 For each $\mu_{0} \in \mathcal{P}_{\exp }$, there exists a map $[0,+\infty) \ni t \mapsto \mu_{t} \in \mathcal{P}_{\exp }$ that solves (3.1). 
Remark 3.10 According to Theorem 3.9, if the initial state of the population is subPoissonian, i.e., $\mu_{0} \in \mathcal{P}_{\text {exp }}$, then the evolution $\mu_{0} \rightarrow \mu_{t}$ preserves this property, and hence the sub-Poissonian decay of the occupation probabilities (2.22). This is true for both long and short repulsion cases mentioned in Remark 3.2 - no matter how short is the repulsion.

The proof of Theorem 3.9 is quite technical and its complete presentation will be done in a separate publication. Here we only outline the main steps. The basic idea is to use (2.19) by which one gets

$$
\mu(L F)=\mu(L K G)=\mu(K \widehat{L} G)=\left\langle\left\langle k_{\mu}, \widehat{L} G\right\rangle\right\rangle=\left\langle\left\langle L^{\dagger} k_{\mu}, G\right\rangle\right.
$$

where the action of $L^{\dagger}$ is obtained from (3.6) by means of (2.20) in the following form

$$
\begin{aligned}
\left(L^{\dagger} k\right)(\gamma)= & \left(\left(B_{1}+B_{2}+B_{3}+B_{4}\right) k\right)(\gamma) \\
= & \sum_{x \in \gamma} E^{+}(x, \gamma \backslash x) k(\gamma \backslash x)+\sum_{x \in \gamma} \int_{X} a^{+}(x-y) k(\gamma \backslash x \cup y) d y \\
& -\left(\sum_{x \in \gamma} E^{-}(x, \gamma \backslash x)\right) k(\gamma)-\int_{X}\left(\sum_{x \in \gamma} a^{-}(x-y)\right) k(\gamma \cup y) d y .
\end{aligned}
$$

The latter is then used in the following evolution equation

$$
\frac{d}{d t} k_{t}=L^{\dagger} k_{t}, \quad k_{0}=k_{\mu_{0}},
$$

where $k_{\mu_{0}}$ is the correlation function of the initial state $\mu_{0} \in \mathcal{P}_{\text {exp. }}$. In fact, (3.14) is an infinite chain of coupled equations involving $k_{t}^{(m)}$ with $m \geq 1$. Its first two members look as follows.

$$
\begin{aligned}
\frac{d}{d t} k_{t}^{(1)}(x)= & b^{+}(x)+\int_{X} a^{+}(x-y) k_{t}^{(1)}(y) d y \\
& -b^{-}(x) k^{(1)}(x)-\int_{X} a^{-}(x-y) k_{t}^{(2)}(x, y) d y \\
\frac{d}{d t} k_{t}^{(2)}(x, y)= & b^{+}(x) k_{t}^{(1)}(y)+b^{+}(y) k_{t}^{(1)}(x)+a^{+}(x-y)\left[k_{t}^{(1)}(x)+k_{t}^{(1)}(y)\right] \\
& +\int_{X}\left[a^{+}(x-u) k^{(2)}(y, u)+a^{+}(y-u) k^{(2)}(x, u)\right] d u \\
& -\left[b^{-}(x)+b^{-}(y)+2 a^{-}(x-y)\right] k_{t}^{(2)} \\
& -\int_{X}\left[a^{-}(x-u)+a^{-}(y-u)\right] k_{t}^{(3)}(x, y, u) d u .
\end{aligned}
$$


To study (3.14) we introduce Banach spaces $\mathcal{K}_{C}$, dual to those introduced in Definition 3.5 , cf. the last equality in (3.12). Namely, for $C>0$ and $k$ as in (2.19), we set

$$
\begin{aligned}
|k|_{C} & =\sup _{m \geq 0} \operatorname{ess} \sup _{\left(x_{1}, \ldots x_{m}\right) \in X}\left|k^{(m)}\left(x_{1}, \ldots, x_{m}\right)\right|, \\
\mathcal{K}_{C} & =\left\{k: \Gamma_{0} \rightarrow \mathbb{R}:|k|_{C}<\infty\right\} .
\end{aligned}
$$

These Banach spaces constitute an ascending scale $\{\mathcal{K}\}_{C>0}$ in the sense that $\mathcal{K}_{C} \hookrightarrow$ $\mathcal{K}_{C^{\prime}}$ for $C^{\prime}>C$, where $\hookrightarrow$ denotes continuous embedding. Similarly as in the proof of Proposition 3.7, one can show that $L^{\dagger}: \mathcal{K}_{C} \rightarrow \mathcal{K}_{C+\varepsilon}$, holding for all positive $C$ and $\varepsilon$. However, this is not enough for constructing solutions of (3.17) which we need. Loosely speaking, the main reason for this is that the sequence of the Picard approximates of such a solution diverges for any $t>0$, which is related to the fact that $B_{1}$ and $B_{3}$ in (3.13) are 'quadratic' in $|\gamma|$, cf. (3.10). What saves the day is the observation that they have different signs, and hence can compensate each other. This is realized by means of the following estimate, which is an adaptation of the one proved in [29, Proposition 2.5].

Proposition 3.11 If the kernels $a^{ \pm}$satisfy Assumption 3.1, then there exist positive $\alpha$ and $\beta$ such that the following holds

$$
\forall \gamma \in \Gamma_{0} \quad \alpha|\gamma|+\sum_{x \in \gamma} \sum_{y \in \gamma \backslash x} a^{-}(x-y) \geq \beta \sum_{x \in \gamma} \sum_{y \in \gamma \backslash x} a^{+}(x-y) .
$$

This estimate reflects the very concept of our approach-to compensate attraction by repulsion. In [20, Assumption 1, page 142], a similar property was imposed as an additional condition, see also [20, Sect. 3.4]. Here we claim that it holds if $a^{-}$is separated away from zero in a ball of an arbitrarily small radius.

For $0<C<C^{\prime}$, by $\mathcal{L}\left(C, C^{\prime}\right)$ we denote the Banach space of bounded linear operators $A: \mathcal{K}_{C} \rightarrow \mathcal{K}_{C^{\prime}}$ equipped with the usual operator norm $\|A\|$. By means of (3.18) we prove the next statement.

Lemma 3.12 For each $C>0$, there exist $C$-dependent $C^{\prime}>C$ and $T_{C}>0$ such that the following holds. There exists a unique continuous map $\left[0, T_{C}\right) \ni t \mapsto U_{C}(t) \in$ $\mathcal{L}\left(C, C^{\prime}\right)$, such that: $(a) U_{C}(0)$ is an embedding operator; (b) for each $t \in\left(0, T_{C}\right)$, there exists $C_{t} \in\left(C, C^{\prime}\right)$ such that $U_{C}(t)$ maps $\mathcal{K}_{C}$ in $\mathcal{K}_{C_{t}} \hookrightarrow \mathcal{K}_{C^{\prime}}$ and the derivative of the map $t \mapsto U_{C}(t) \in \mathcal{L}\left(C, C^{\prime}\right)$ satisfies

$$
\frac{d}{d t} U_{C}(t)=L^{\dagger} U_{C}(t)
$$

where $L^{\dagger}$ is considered as a bounded linear operator from $\mathcal{K}_{C_{t}}$ to $\mathcal{K}_{C^{\prime}}$.

Corollary 3.13 It follows that $k_{t}=U_{C}(t) k_{0}$ is a solution of (3.14).

The proof of Lemma 3.12 follows mostly along the line of arguments used in the proof of analogous statements [20, Lemma 4.5] and [29, Lemma 5.4]. The choice of $C^{\prime}$ depends on $C$ and $\beta$ in (3.18), as well as on the bounds that appear in (3.10) and 
(3.11). The dependence of $T_{C}$ on $C$ can be calculated explicitly. Unfortunately, this dependence is such that the continuation of the solution $k_{t}$ to all $t>0$ is impossible. Moreover, this solution need not be the correlation of any $\mu_{t} \in \mathcal{P}(\Gamma)$ and hence cannot be used to construct solutions of (3.1). Even the positivity of $k_{t}$, cf. (2.13), cannot be secured. The latter drawbacks are eliminated in the next statement. Let $\mathcal{K}_{C}^{\star}$ stand for the subset of $\mathcal{K}_{C}$ such that each $k \in \mathcal{K}_{C}^{\star}$ is the correlation function of some $\mu$. Note that this $\mu$ is unique, cf. [29, Proposition 2.3]. By (2.13) $k$ is positive, by (3.17) it follows that $\mu \in \mathcal{P}_{\exp }$ and its type is at most $C$.

Lemma 3.14 Let $U_{C}(t)$ be as in Lemma 3.12. Then, for each $t \in\left[0, T_{C}\right)$, it follows that $U(t): \mathcal{K}_{C}^{\star} \rightarrow \mathcal{K}_{C^{\prime}}^{\star}$

The proof of this statement is based on a refined construction, similar to that used in the proof of [29, Lemma 6.1]. One of its outcomes is the positivity of $k_{t}$, which we then employ to prove that $U_{C}(t)$ maps $\mathcal{K}_{C}$ into $\mathcal{K}_{C^{\prime \prime}}$ with $C^{\prime \prime}$ essentially smaller than $C^{\prime}$ mentioned in Lemma 3.12. This allows us to construct continuations of the solution $k_{t}$ to all $t>0$ by a method similar to that used in [29, Sect. 7]. All these aspects are summarized in the following statement, in which by a classical solution of (3.14) we mean a map $t \mapsto k_{t}$ which is continuously differentiable in the corresponding Banach spaces and satisfy both equalities mentioned therein.

Lemma 3.15 For each $C>0$, there exists a map $\mathbb{R}_{+} \ni t \mapsto C_{t}$ such that, for all $\mu_{0} \in \mathcal{P}_{\exp }$ of type $\leq C$, the equation (3.14) with $k_{0}=k_{\mu_{0}}$ has a unique classical solution $k_{t} \in \mathcal{K}_{C_{t}}^{\star}$.

Proof of Theorem 3.9 Since $k_{t}$ mentioned in Lemma 3.15 is a classical solution, for $G \in \mathcal{G}$ we have

$$
\frac{d}{d t}\left\langle\left\langle k_{t}, G\right\rangle\right\rangle=\left\langle\left\langle\frac{d}{d t} k_{t}, G\right\rangle\right\rangle=\left\langle\left\langle L^{\dagger} k_{t}, G\right\rangle .\right.
$$

Then the solution of (3.1) which we aim to get can be defined by its values on $\mathcal{D}(L)$, cf. Proposition 3.3 and (3.9), as follows

$$
\mu_{t}(F)=\mu_{t}(K G)=\left\langle\left\langle k_{t}, G\right\rangle\right\rangle
$$

which by (3.20) yields

$$
\begin{aligned}
\int_{0}^{t} \mu_{u}(L F) d u & =\int_{0}^{t} \mu_{u}(L K G) d u=\int_{0}^{t}\left\langle\left\langle k_{u}, \widehat{L} G\right\rangle d u\right. \\
& =\int_{0}^{t}\left\langle\left\langle L^{\dagger} k_{u}, G\right\rangle\right\rangle d u=\left\langle\left\langle k_{t}, G\right\rangle-\left\langle\left\langle k_{0}, G\right\rangle=\mu_{t}(F)-\mu_{0}(F),\right.\right.
\end{aligned}
$$

which completes the proof. 


\subsection{The kinetic equation and beyond}

According to Definition 2.2, sub-Poissonian states have finite correlations, and their correlation measures (2.6) are absolutely continuous with respect to the corresponding Lebesgue measures, cf. (2.10) and (2.12). That is, each $\mu \in \mathcal{P}_{\exp }$ is completely characterized by its correlation function $k_{\mu}$. The Poisson measures from $\mathcal{P}_{\exp }$ are then characterized by densities $q \in L^{\infty}(X)$, see (2.14). At the mesoscopic level obtained by a scaling procedure, see $[17,26]$, the corpuscular structure of the system is lost, i.e., it appears now as a continuous medium characterized solely by its density, the evolution of which is now the evolution of the whole system. The inter-particle interactions give rise to a state-dependent modulation of the environment, which corresponds to the mean-field approach widely used in statistical physics [38].

Without mathematical justification-i.e., without an explicit passage to the mesoscopic scaling limit-kinetic equations are derived by the so called moment closure (decoupling) procedure [31]. Basing on the results of [17,26], we can state here that the kinetic equation corresponding to our model is obtained from the first equation (3.15) of the chain encrypted in (3.14) by 'decoupling' $k_{t}^{(2)}(, y) \rightarrow k_{t}^{(1)}(x) k_{t}^{(1)}(y)$. This turns the infinite chain into a single nonlinear equation

$$
\begin{aligned}
\frac{d}{d t} q_{t}(x)= & b^{+}(x)-b^{-}(x) q_{t}(x) \\
& +\int_{X} a^{+}(x-y) q_{t}(y) d y-q_{t}(x) \int_{X} a^{-}(x-y) q_{t}(y) d y,
\end{aligned}
$$

where $q_{t}=k_{t}^{(1)}$ is the density in question, satisfying the initial condition $\left.q_{t}\right|_{t=0}=$ $k_{\mu_{0}}^{(1)}$. Despite the mentioned loss of information, such equations can provide much more detailed description of the system's evolution. They are pretty well accessible to numerical methods, especially tailored to study such models [27,35]. We plan to realize this in a forthcoming paper. Note that the sub-Poissonicity established in Theorem 3.9 'justifies' passing to the description of the evolution of the considered system based on the kinetic equation.

The moment closure procedure can also be done at the next level, i.e., applied to $k_{t}^{(3)}$ in (3.16). This allows one to go beyond the mean-field description, see [34] where it was performed for the Bolker-Pacala model with the help of the Kirkwood superposition approximation [4]. We plan to realize this as well, in particular, we expect to clarify the peculiarities of the cases mentioned in Remark 3.2.

Funding By Narodowe Centrum Nauki (NCN), Poland, grant 2017/25/B/ST1/00051.

\section{Declarations}

Conflict of interest The author(s) declare that they have no conflict of interest.

Availability of data and material Data sharing not applicable to this article as no datasets were generated or analyzed during the current study. 
Code availability Not applicable.

Open Access This article is licensed under a Creative Commons Attribution 4.0 International License, which permits use, sharing, adaptation, distribution and reproduction in any medium or format, as long as you give appropriate credit to the original author(s) and the source, provide a link to the Creative Commons licence, and indicate if changes were made. The images or other third party material in this article are included in the article's Creative Commons licence, unless indicated otherwise in a credit line to the material. If material is not included in the article's Creative Commons licence and your intended use is not permitted by statutory regulation or exceeds the permitted use, you will need to obtain permission directly from the copyright holder. To view a copy of this licence, visit http://creativecommons.org/licenses/by/4.0/.

\section{References}

1. Banasiak, J., Lachowicz, M., Moszyński, M.: Semigroups for generalized birth-and-death equations in $\ell^{p}$ spaces. Semigroup Forum 73, 175-193 (2006)

2. Bellomo, N., Brezzi, F.: Mathematics, complexity and multiscale features of large systems of selfpropelled particles. Math. Models Methods Appl. Sci. 26, 207-214 (2016)

3. Bellomo, N., Knopoff, D., Soler, J.: On the difficult interplay between life, "complexity", and mathematical sciences. Math. Models Methods Appl. Sci. 23, 1861-1913 (2013)

4. Ben-Naim, A.: The Kirkwood superposition approximation, revisited and reexamined. J. Adv. Chem. 1, 27-35 (2013)

5. Berestycki, N., Etheridge, A.M., Hutzenthaler, M.: Survival, extinction and ergodicity in a spatially continuous population model. Markov Process. Related Fields 15, 265-288 (2009)

6. Bogachev, V.I., Krylov, N.V., Röckner, M., Shaposhnikov, S. V.: Fokker-Planck-Kolmogorov Equations, Mathematical Surveys and Monographs, 207. American Mathematical Society, Providence, RI (2015)

7. Bolker, B.M., Pacala, S.W.: Using moment equations to understand stochastically driven spatial pattern formation in ecological systems. Theoret. Population Biol. 52, 179-197 (1997)

8. Bolker, B.M., Pacala, S.W., Neuhauser, C.: Spatial dynamics in model plant communities: What do we really know? Am. Nat. 162, 135-148 (2003)

9. Boyadziev, K. N.: Exponential polynomials, Stirling numbers, and evaluation of some gamma integrals, Abstr. Appl. Analysis 168672, 18 (2009)

10. de Bruijn, N.G.: Asymptotic Methods in Analysis, 3rd edn. Dover Publishers Inc., New York (1981)

11. Cox, J.T.: Coalescing random walks and voter model consensus times on the torus in $\mathbb{Z}^{d}$. Ann. Probab. 17, 1333-1366 (1989)

12. Daley, D. J., Vere-Jones, D.: An Introduction to the Theory of Point Processes. Vol. I. Elementary Theory and Methods. Second edition. Probability and its Applications (New York). Springer-Verlag, New York (2003)

13. Dawson, D. A.: Measure-Valued Markov Processes. École d'Été de Probabilités de Saint-Flour XXI1991, 1-260, Lecture Notes in Math., 1541, Springer, Berlin (1993)

14. Dobrushin, R. L., Sinai, Y. G., Sukhov, Y. M.: Dynamical systems of statistical mechanics, in Dynamical Systems II. Encyclopaedia of Mathematical Sciences, vol 2., Y. G. Sinai, eds, Springer, Berlin, Heidelberg (1989)

15. Ethier, S.N., Kurtz, T.G.: Markov Processes: Characterization and Convergence. Wiley, New York (1986)

16. Finkelshtein, D.L., Kondratiev, Y.G., Kutovyi, O.: Individual based model with competition in spatial ecology. SIAM J. Math. Anal. 41, 297-317 (2009)

17. Finkelshtein, D.L., Kondratiev, Y.G., Kozitsky, Yu., Kutovyi, O.: The statistical dynamics of a spatial logistic model and the related kinetic equation. Math. Models Methods Appl. Sci. 25, 343-370 (2015)

18. Jasińska, D., Kozitsky, Yu.: Dynamics of an infinite age-structured particle system, Math. Methods Appl. Sci. https://doi.org/10.1002/mma.7174 (2021)

19. Kingman, J. F. C.: Poisson processes. Oxford Studies in Probability, 3. Oxford Science Publications. The Clarendon Press, Oxford University Press, New York (1993)

20. Kondratiev, Y.G., Kozitsky, Yu.: The evolution of states in a spatial population model. J. Dyn. Diff. Equat. 30, 135-173 (2018) 
21. Kondratiev, Yu., Kozitsky, Yu.: Self-regulation in the Bolker-Pacala model. Appl. Math. Lett. 69, 106-112 (2017)

22. Kondratiev, Yu., Kozitsky, Yu.: Evolution of states in a continuum migration model. Anal. Math. Phys. 8, 93-121 (2018)

23. Kondratiev, Yu., Kozitsky, Yu., Shoikhet, D.: Dynamical systems on sets of holomorphic functions. In: Agranovsky, M., et al. (eds.) Contemporary Mathematics, 553. Amer. Math. Soc, RI (2011)

24. Kozitsky, Yu.: Dynamics of spatial logistic model: finite systems, In: Semigroups of Operators - Theory and Applications: Będlewo, Poland, October 2013, Banasiak, J et al (eds), Springer Proceedings in Mathematics \& Statistics 113, Springer (2015)

25. Kozitsky, Yu.: Stochastic branching at the edge: Individual-based modeling of tumor cell proliferation. J. Evol. Equat. 21, 2081-2104 (2021)

26. Kozitsky, Yu.: Evolution of infinite populations of immigrants: micro- and mesoscopic description. J. Math. Anal. Appl. 477, 294-333 (2019)

27. Kozitsky, Yu., Omelyan, I., Pilorz, K.: Jumps and coalescence in the continuum: A numerical study. Appl. Math. Comput. 390, 125610 (2021)

28. Kozitsky, Yu., Tanaś, A.: Self-regulation in infinite populations with fission-death dynamics. Phys. Lett. A 382, 2455-2458 (2018)

29. Kozitsky, Yu., Tanaś, A.: Evoluition of states of an infiniote fission-death system. J. Math. Anal. Appl. 125222, (2021)

30. Lenard, A.: Correletion functions and the uniqueness of the state in classical statistical mechanics. Comm. Math. Phys. 30, 35-44 (1973)

31. Murrell, D.J., Dieckmann, U., Law, R.: On moment closures for population dynamics in contunuous space. J. Theoret. Biol. 229, 421-432 (2004)

32. Neuhauser, C.: Mathematical challenges in spatial ecology. Notices AMS 48(11), 1304-1314 (2001)

33. North, A., Ovaskainen, O.: Interactions between dispersal, competition, and landscape heterogeneity. Oikos 116, 1106-1119 (2007)

34. Omelyan, I., Kozitsky, Yu.: Spatially inhomogeneous population dynamics: beyond the mean field approximation. J. Phys. A. Math. Theor. 52, 305601 (2019)

35. Omelyan, I., Kozitsky, Yu., Pilorz, K.: Algorithm for numerical solutions to the kinetic equation of a spatial population dynamics model with coalescence and repulsive jumps. Numer. Algor. (2020). https://doi.org/10.1007/s11075-020-00992-9

36. Riordan, J.: Combinatorial Identities. John Wiley \& Sons Inc, New York - London - Sydney (1968)

37. Ruelle, D.: Superstable interactions in classical statistical mechanics. Comm. Math. Phys. 18, 127-159 (1970)

38. Simon, B.: The Statistical Mechanics of Lattice Gases. Princeton University Press, Pronceton NJ (1993)

Publisher's Note Springer Nature remains neutral with regard to jurisdictional claims in published maps and institutional affiliations. 